\title{
RESEARCH
}

Open Access

\section{Comparison of implant placement accuracy in two different preoperative digital workflows: navigated vs. pilot-drill-guided surgery}

Johannes Spille ${ }^{1 *+}$, Feilu Jin ${ }^{2+}$, Eleonore Behrens ${ }^{1}$, Yahya Açil ${ }^{1}$, Jürgen Lichtenstein ${ }^{1}$, Hendrik Naujokat ${ }^{1}$, Aydin Gülses ${ }^{1}$, Christian Flörke $^{1+}$ and Jörg Wiltfang ${ }^{1+}$

\begin{abstract}
Background: The aim of the study is to evaluate the accuracy of a new implant navigation system on two different digital workflows.

Methods: A total of 18 phantom jaws consisting of hard and non-warping plastic and resembling edentulous jaws were used to stimulate a clinical circumstance. A conventional pilot-drill guide was conducted by a technician, and a master model was set by using this laboratory-produced guide. After cone beam computed tomography (CBCT) and 3D scanning of the master models, two different digital workflows (marker tray in CBCT and 3D-printed tray) were performed based on the Digital Imaging Communication in Medicine files and standard tessellation language files. Eight Straumann implants $(4.1 \mathrm{~mm} \times 10 \mathrm{~mm})$ were placed in each model, six models for each group, resulting in 144 implant placements in total. Postoperative CBCT were taken, and deviations at the entry point and apex as well as angular deviations were measured compared to the master model.
\end{abstract}

Results: The mean total deviations at the implant entry point for MTC (marker tray in CBCT), 3dPT (3d-printed tray), and PDG (pilot-drill guide) were $1.024 \pm 0.446 \mathrm{~mm}, 1.027 \pm 0.455 \mathrm{~mm}$, and $1.009 \pm 0.415 \mathrm{~mm}$, respectively, and the mean total deviations at the implant apex were $1.026 \pm 0.383 \mathrm{~mm}, 1.116 \pm 0.530 \mathrm{~mm}$, and $1.068 \pm 0.384 \mathrm{~mm}$. The angular deviation for the MTC group was $2.22 \pm 1.54^{\circ}$. The $3 \mathrm{dPT}$ group revealed an angular deviation of $1.95 \pm$ $1.35^{\circ}$, whereas the PDG group showed a mean angular deviation of $2.67 \pm 1.58^{\circ}$. Although there were no significant differences among the three groups $(P>0.05)$, the navigation groups showed lesser angular deviations compared to the pilot-drill-guide (PDG) group. Implants in the 3D-printed tray navigation group showed higher deviations at both entry point and apex.

Conclusions: The accuracy of the evaluated navigation system was similar with the accuracy of a pilot-drill guide. Accuracy of both preoperative workflows (marker tray in CBCT or 3D-printed tray) was reliable for clinical use.

Keywords: Navigation, Accuracy, Implant system, Digital workflow

\footnotetext{
* Correspondence: johannesheinrich.spille@uksh.de

${ }^{\dagger}$ Johannes Spille, Feilu Jin, Christian Flörke and Jörg Wiltfang contributed equally to this work.

${ }^{1}$ Christian Albrechts University, Department of Oral and Maxillofacial Surgery, University Hospital of Schleswig-Holstein, Campus Kiel, Arnold-Heller-Straße 3, 24105 Kiel, Germany

Full list of author information is available at the end of the article
}

\section{Springer Open}

(c) The Author(s). 2021 Open Access This article is licensed under a Creative Commons Attribution 4.0 International License, which permits use, sharing, adaptation, distribution and reproduction in any medium or format, as long as you give appropriate credit to the original author(s) and the source, provide a link to the Creative Commons licence, and indicate if changes were made. The images or other third party material in this article are included in the article's Creative Commons licence, unless indicated otherwise in a credit line to the material. If material is not included in the article's Creative Commons licence and your intended use is not permitted by statutory regulation or exceeds the permitted use, you will need to obtain permission directly from the copyright holder. To view a copy of this licence, visit http://creativecommons.org/licenses/by/4.0/. 


\section{Background}

Precise placement of implants is of great importance in survival of an implant-supported prosthesis [1]. Implant malposition was reported to be the most likely reason to cause peri-implantitis. It has been proclaimed that approximately $50 \%$ of all peri-implantitis cases had been triggered by implant malposition during surgery [2]. To achieve the optimal functional and esthetic results, the concept of "guided surgery" was introduced to allow clinicians to achieve higher precision of dental implant placement $[3,4]$. Nowadays, a guided surgery could be performed by using either surgical guides or dynamic navigation systems. Although computer-aided implant systems have been proved to improve the accuracy in either in vivo or in vitro studies $[5,6]$, a conventional surgical template is still the first choice to many surgeons, considering the cost and complexity of computeraided guidance [7].

A conventional surgical guide is known to be fabricated on a gypsum cast by the technician, which is still the most frequently used in clinical circumstances, followed by computer-aided design/computer-aided manufacturing (CAD/CAM) static guidance [8].

Although multiple studies have proved the use of surgical guides could improve the accuracy of final implant position $[9,10]$, certain limitations should still be considered such as poor visualization of anatomical structures, poor bone cooling during osteotomy, a possibility of loosening the template during drilling, and problems related to the intraoperative modification of the preoperative plan [11-13].

In recent years, dynamic navigation systems were developed to find a better solution to solve these problems. Supported by a computed-navigation system, preplanned position of implant and real-time position of the drill tip could be easily detected on a screen [12]. Therefore, suitable adjustment can be made and a more precise placement could be ensured [13].

A digital plan based on preoperatively marked cone beam computed tomography (CBCT) images is a widely accepted planning modality by both clinicians and researchers. However, an intraoral scanning to gain the standard tessellation language (STL) files followed by a 3D-printed tray seems to be also promising [14].

The aim of the current study was to assess the accuracy of implant placement using a new navigation system in comparison with $\mathrm{CBCT}$ (MTC) and 3D-printed tray (3dPT) workflows.

\section{Material and methods}

\section{Study design}

On Frasaco models, which have exhibited the same clinical situation, the following three insertion protocols were established:
- Pilot-drill guided

- Marker tray in CBCT navigation

- 3D-printed tray navigation

A single researcher has planned the locations of the implants in the jaw prior to experiments. In order to avoid possible bias, all implant insertions were performed by another single, blinded researcher for all groups, who was not involved in the evaluation process of the accuracy. Another researcher, who was blinded to the preoperative data plan, has conducted the postoperative DVT scans and determined the positions of the inserted implants compared to the master typodont. The calculation of the accuracy values was then carried out automatically using selected computer programs. All results were included in this study; no results were discarded or recalculated.

The study evaluates the accuracy of implant placement in Frasaco models under instruction from the Denacam System of the company mininavident AG (Liestal, Schweiz). Denacam is a dynamic computer-assisted surgical system and uses the principles of stereo triangulation from optical cameras. As a real-time navigation system, Denacam uses a small, prefabricated intraoral marker to coordinate the planned implant position and the real-time position of the drill during the operation. The practician recognizes during the operation deviations in the entry point, the apex, and the angle on a screen. In this way, the current drill position and the planned implant insertion can be coordinated.

\section{Fabrication of the surgical guide}

Partially edentulous Frasaco mandible (Frasaco $\mathrm{GmbH}$, Tettnang, Germany) was used as the master typodont (Fig. 1). Eleven teeth on the jaw were missing in total, and four teeth on each side were decided to be replaced with implants, which made eight implants in total. A wax-up prothesis was firstly accomplished to mimic an ideal denture on a duplicated cast model. Then, the final acrylic-made pilot-drill guide was fabricated based on the wax prosthesis (Fig. 2). Eight Steco titanium drill sleeves $(2.2 \mathrm{~mm}$ inner, $3.5 \mathrm{~mm}$ outer, and $5.0 \mathrm{~mm}$ in length; Steco-system-technik GmbH\&Co.KG) corresponding to planned implant positions were inserted into the guide to guarantee a more stable guidance.

\section{Preparation of the master typodont}

The drillings on the master typodont were firstly guided by the pilot-drill guide, and as for the twist drills, parallel pins in neighboring drill holes were used to maintain proper angles. Eight Straumann bone level implants (4.1 $\mathrm{mm} \times 10 \mathrm{~mm}$; Straumann Holding AG, Switzerland) were placed manually into the implant beds after osteotomies. 


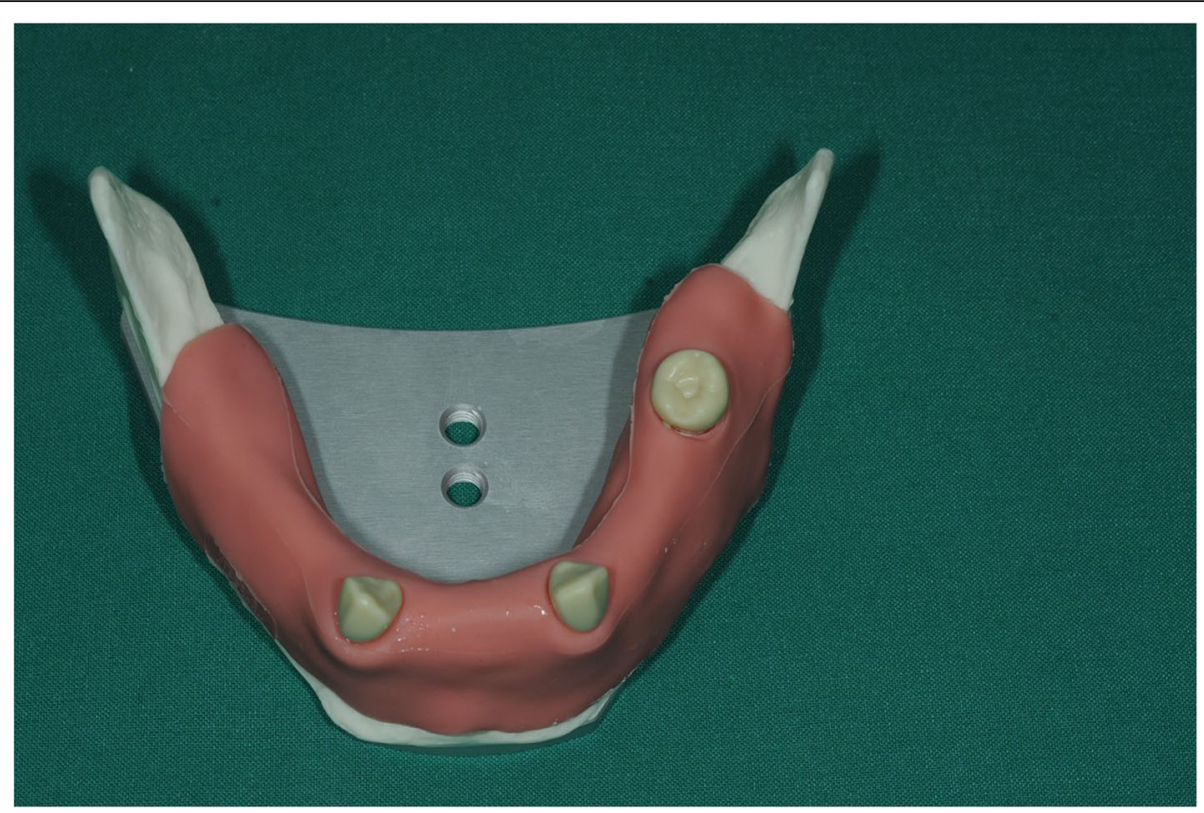

Fig. 1 Original Frasaco mandible

\section{Workflow for the marker tray in CBCT navigation (MTCN)} group

To register the jaw into the navigation system, a marker tray with the registration marker on was firmly adapted to the master typodont's left ramus by using vinylpolysiloxane impression material (Flexitime, KULZER GmbH) (Fig. 3). Usually, this type of system has a registration marker, which should be fixed on the remaining teeth at the same dental arch during the implant insertion. In the current study, the marker tray was placed on the left ascending ramus; thus, a total of eight implants should be placed. The re-positioning of the marker could negatively influence the accuracy of the measurement. The master typodont was then scanned by CBCT machine (KaVo 3D eXam, resolution: 0.2 voxel), and the Digital Imaging Communication in Medicine (DICOM) files were imported into the coDiagnostiX system (Dental wings $\mathrm{GmbH}$, Chemnitz, Germany). The geometry of the implants was

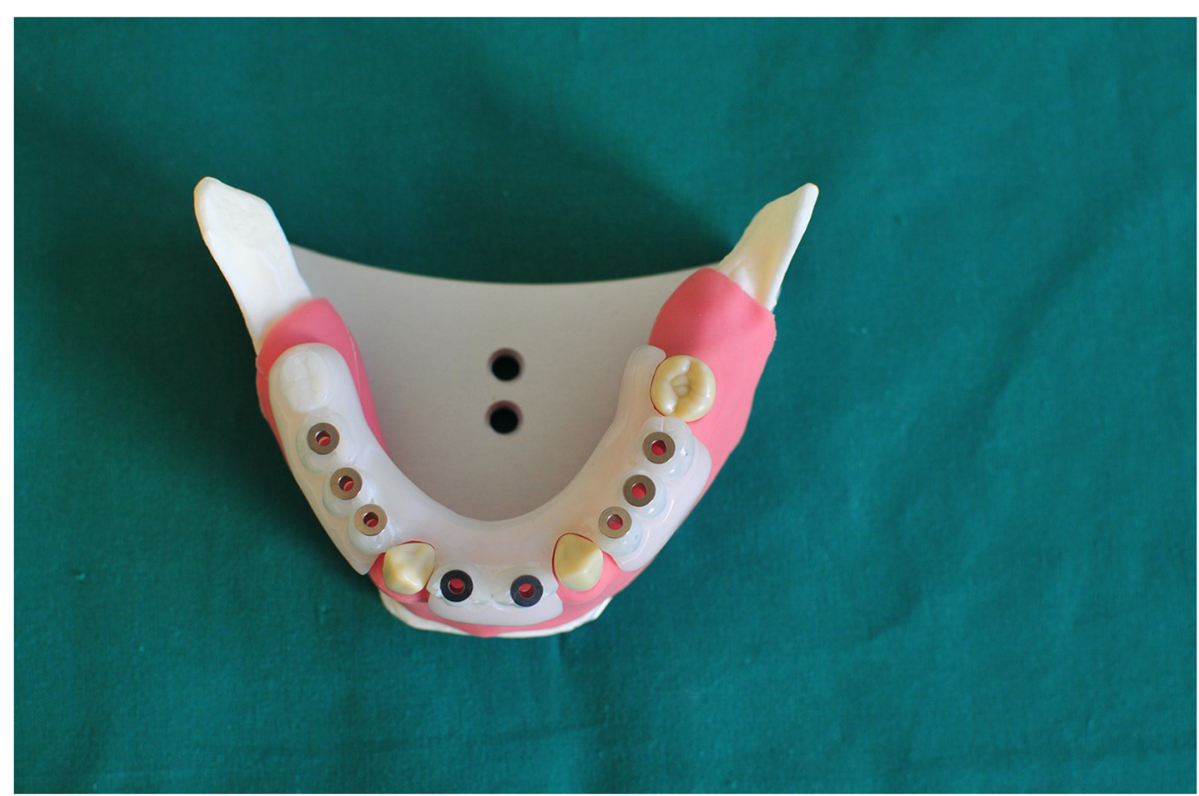

Fig. 2 Frasaco jaw with pilot-drill guide on 


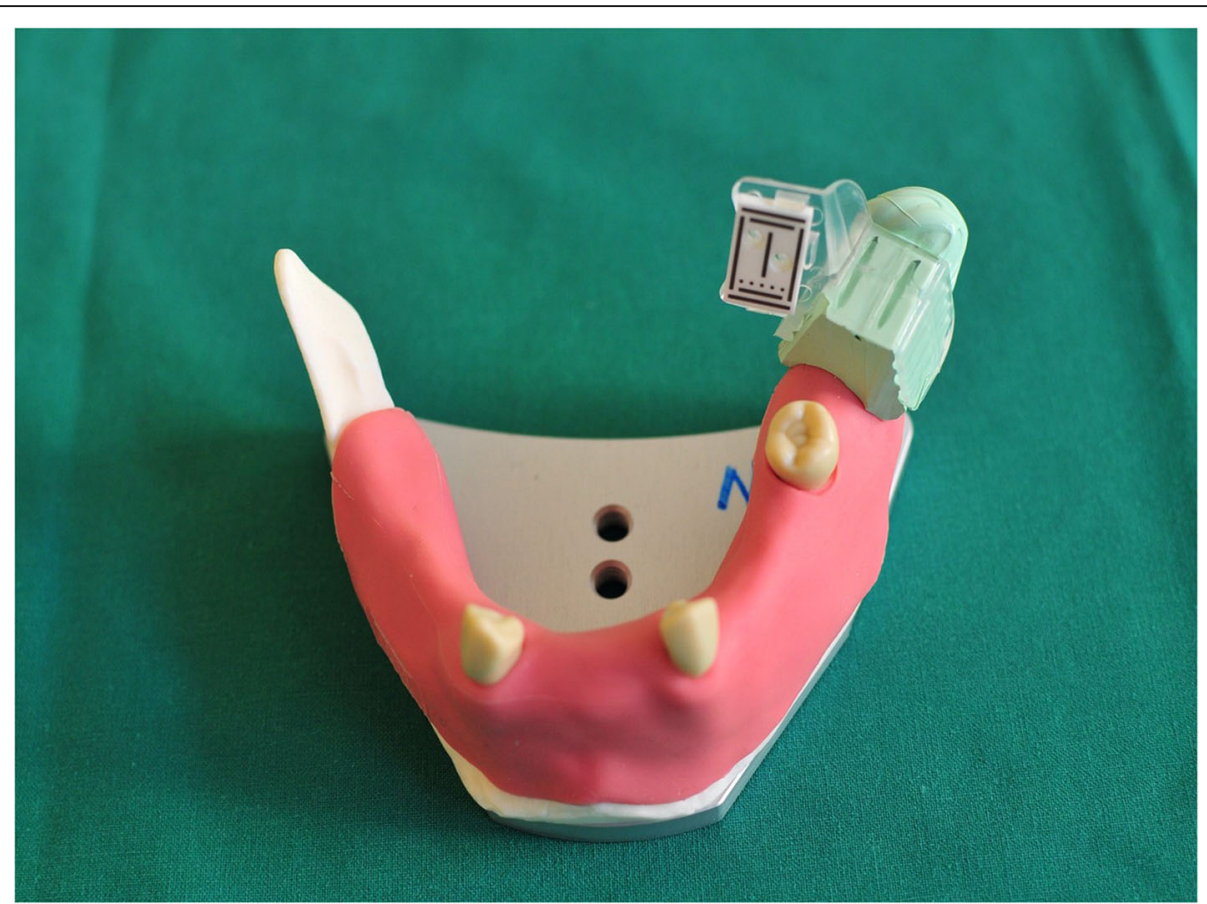

Fig. 3 Frasaco jaw with marker tray on

easily recognized, and the virtual implants were located more precisely by superimposing upon the images. The digital plan was imported into the navigation system afterwards. The slice thickness of the CBCT machine was set to 0.2 voxel on application of Denacam System, which had no significant effect on the clinical application.
Workflow for the 3D-printed tray navigation (3dPTN) group

Both the master typodont with original marker tray on and the particular Frasaco typodont which was chosen for the 3dPTN group were scanned by Shining 3D (Shining 3D Tech. Co. Ltd. Hangzhou, China). The STL files obtained were imported into coDiagnostiX, and

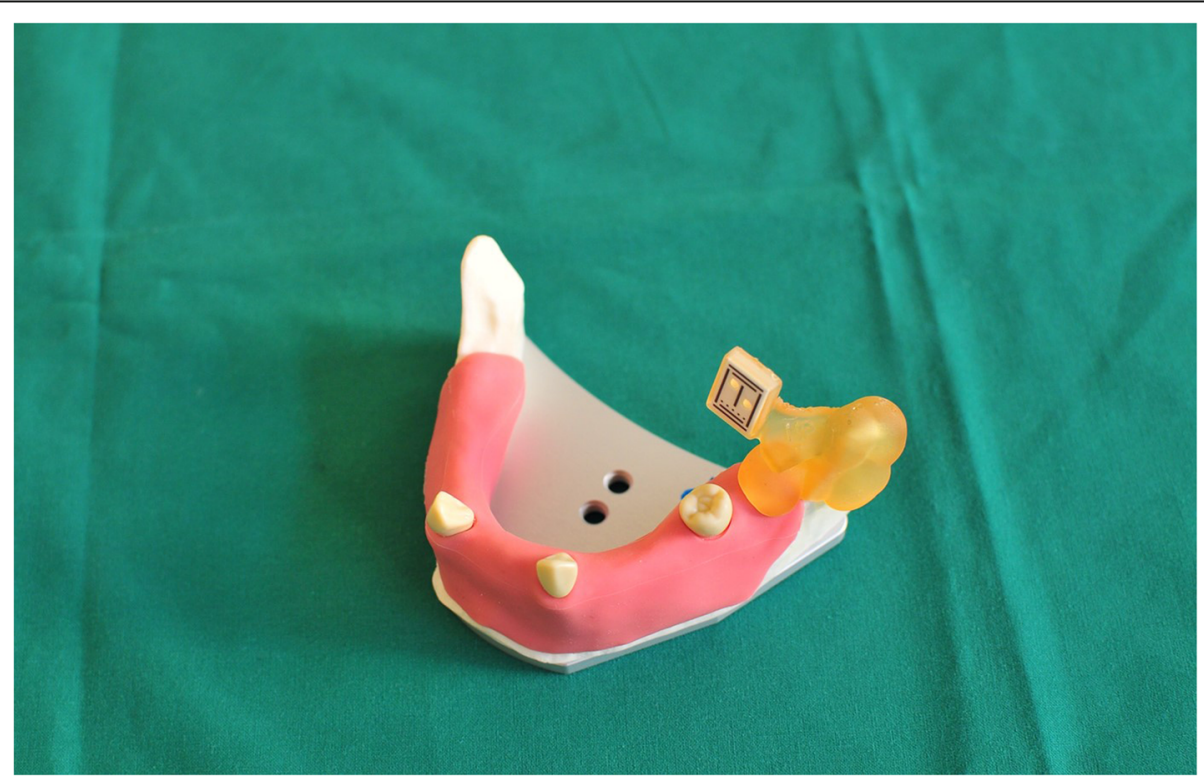

Fig. 4 Frasaco jaw with 3D-printed maker tray on 
three match regions on both typodonts were chosen to do the superimposition, thus, exact position of the marker on master typodont was copied to the $3 \mathrm{dPTN}$ model. The STL model of the tray and the marker provided by the same company as the navigation system was superimposed onto the original tray. New STL files of the $3 \mathrm{dPTN}$ model with the virtual marker tray in the same position as the master typodont were created. The new STL files were then imported to a 3D-printing software, and the newly designed tray was printed in resin by a Form 2 printer (Formlabs Boston Experience, USA, thickness of layer: $0.1 \mathrm{~mm}$ ) (Fig. 4).

\section{Surgical protocol}

The typodont was fixed in a Frasaco phantom head before surgery (Figs. 5 and 6). The soft silicone tissue was then prepared, and the alveolar ridge was exposed. Both the laboratory guide group and the navigation groups followed the standard Straumann drill protocol: a 1.4- $\mathrm{mm}$ round burr was set to define the entry point firstly. A $2.2-\mathrm{mm}$ pilot drill followed by $2.8-\mathrm{mm}$ and $3.5-\mathrm{mm}$ twist drills, except for the navigation groups, was used. Eight Straumann implants with the same size as the master typodont were placed manually in each typodont.

Postoperative $\mathrm{CBCT}$ of each typodont was taken, and the bore holes were refilled with a special material (A-J OP UK K, Frasaco GmbH, Tettnang, Germany). The recemented typodonts were then used for the next trial. Six trials for each group were performed, and DICOM files were obtained for further measurements.

\section{Pilot-drill guided group}

The surgical guide was seated properly during the whole drilling procedure. After the pilot-drill drilling, the surgical guide was removed, and the twist drills were guided by parallel pins. The laser marker on the drill was used to guide the depth.

\section{Marker tray in CBCT navigation group}

The same registration tray used for preoperative $\mathrm{CBCT}$ scan was seated firmly on Frasaco ramus, and each burr used was registered before drilling. If the camera was rotated for a better detection of the marker, the burr should be registered again afterwards.

\section{D-printed tray navigation group}

The printed marker tray was seated on the left ramus as well. The same registration procedure and drilling protocol was followed.

\section{Measurements of error}

Postoperative DICOM files of master typodont as well as one experimental typodont were imported into the Brainlab planning software (version 3.1; BrainlabAG, Munich, Germany). The rigid metal base of the typodont was used to do image fusion, and a spy glass function was used to check (Fig. 7). Once the images were fused approvingly, a linked view would be shown in double windows format.

The entry point at implant shoulder and the apex of each implant were defined on a sagittal view first, and a virtual axial line was drawn. Distances between entry points or apexes were considered to be total

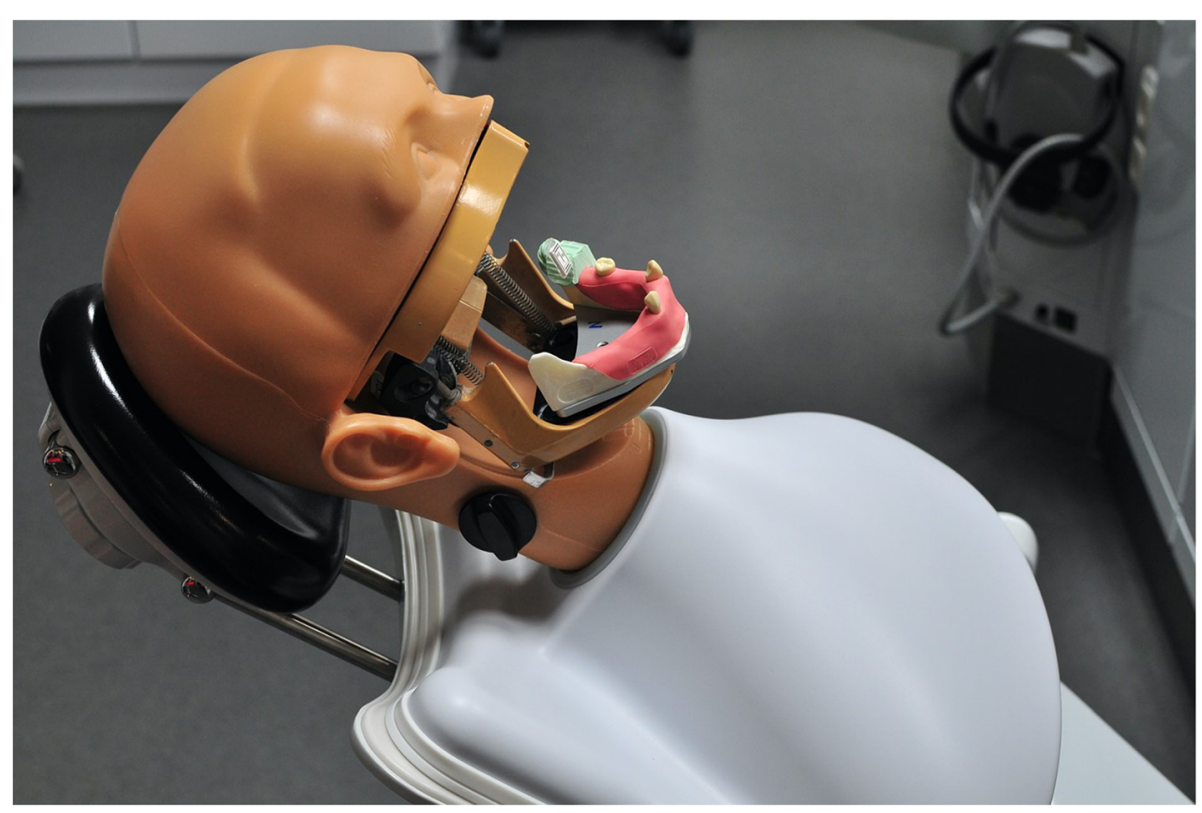

Fig. 5 Frasaco jaw on a phantom head 


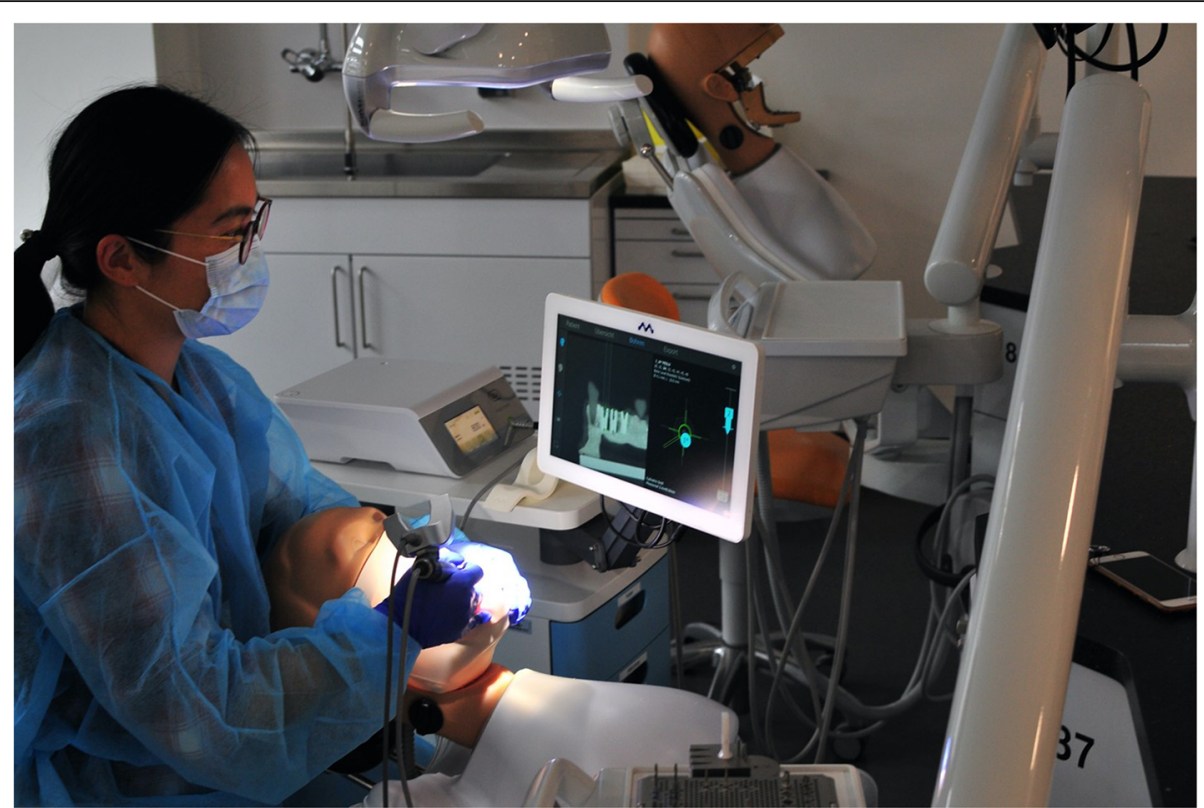

Fig. 6 During the operation

error, and deviations on axial view were defined as lateral errors which represented a mesial-distal deviation and buccal-lingual deviation combined. Error of depth was calculated using the square root formula. The open angle function was used to measure angular error between two virtual axial lines.

\section{Statistical analysis}

SPSS software package (IBM SPSS Statics, version 26.0, IBM) was used, and homogeneity of each parameter among the three groups was checked firstly by means of
Leven's test. All the seven parameters turned out to be homogenous. Then, the one-way analysis of variance (ANOVA) using Scheffe's test as a post-hoc analysis was carried out to analyze the differences among the three groups. $P$ value $<0.05$ was considered to be statistically significant.

\section{Results}

A total of 144 implants ( $n=48$ for each group) were inserted. The mean values and standard deviations of each parameter were established in Table 1. Although

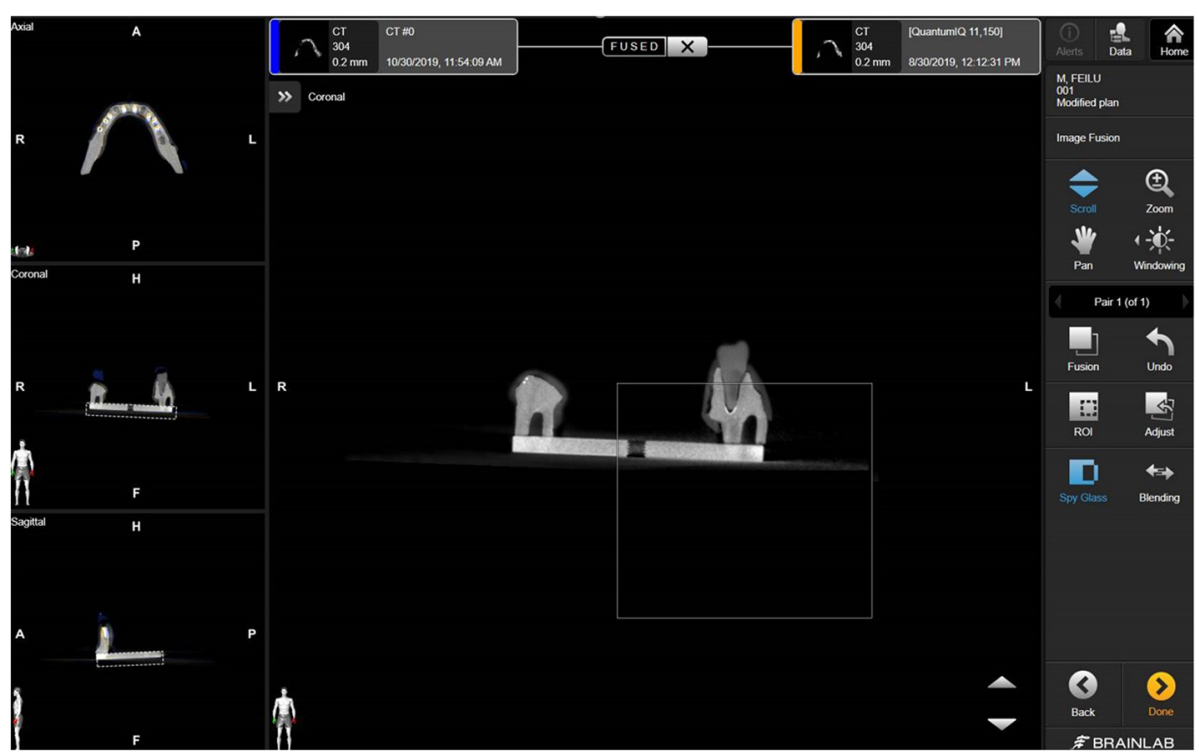

Fig. 7 CBCT images of test model and master model were fused based on the metal base 
Table 1 Mean values and standard deviations of each parameter

\begin{tabular}{llll}
\hline Groups & Total error at entry point (mean \pm SD) & Total error at apex (mean \pm SD) & Angular error (mean \pm SD) \\
\hline Pilot-drill guide & $1.009 \pm 0.415 \mathrm{~mm}$ & $1.068 \pm 0.384 \mathrm{~mm}$ & $2.67 \pm 1.58^{\circ}$ \\
Navigation1 (marker tray in CBCT) & $1.024 \pm 0.446 \mathrm{~mm}$ & $1.026 \pm 0.383 \mathrm{~mm}$ & $2.22 \pm 1.54^{\circ}$ \\
Navigation2 (3D-printed tray) & $1.027 \pm 0.455 \mathrm{~mm}$ & $1.116 \pm 0.530 \mathrm{~mm}$ & $1.95 \pm 1.35^{\circ}$ \\
\hline
\end{tabular}

there were no significant differences among the three groups $(P>0.05)$, the navigation groups showed lower angular deviations compared to the pilot-drill guide (PDG) group $\left(2.67 \pm 1.58^{\circ}\right)$, which were $1.95 \pm 1.35^{\circ}$ for the $3 \mathrm{dPTN}$ group and $2.22 \pm 1.54^{\circ}$ for the MTCN group, respectively. Besides, the $3 \mathrm{dPTN}$ group showed the greatest deviations at both entry point and apex. The PDG group established the best result of total error at entry point, which was $1.009 \pm 0.415 \mathrm{~mm}$, followed by $1.024 \pm 0.446 \mathrm{~mm}$ for the MTCN group and $1.027 \pm$ $0.455 \mathrm{~mm}$ for the 3dPTN group, respectively (Fig. 8).

\section{Discussion}

In this study, attachment of the optical camera put extraweight on the handpiece, which would make the operator lose dexterity when firstly use the equipment. That might be the reason why errors at entry point for the navigation groups were greater than the PDG group. Moreover, angular deviations for the navigation groups were lower than the PDG group, which could be attributed to the modification of the drilling angles and depth based on the real-time feedback on the screen. As for the PDG group, depth control depended on the laser marker on the drill and angular adjustment was less likely to happen once the surgeon started drilling. Chen et al. (2018) reported similar results using a different navigation system on models; total error at entry point was $1.07 \pm 0.48 \mathrm{~mm}$, total error at apex was $1.35 \pm 0.55$ $\mathrm{mm}$, and angular error was $4.45 \pm 1.97^{\circ}$ [15]. Jorba-
Garcia et al. (2019) and Sun et al. (2018) also reported approximate results $[16,17]$. With regard to limited studies of navigation systems, an accuracy of 1-2 mm in vitro has been reported generally [18]. Besides that, discrepancies were usually smaller in in vitro studies than those in clinical studies, considering the disturbing factors like bad vision, movement of patients, and limited operating time [19]. Dental implant surgery uses global technological advances to enable the best possible dental care [20]. For example, there are first approaches of robotic surgery, which have been proven for both simple and complex clinical cases [21].

Moreover, this study shows that it was sometimes quite difficult to rotate the camera to a proper position to get a whole vision of the marker tray, especially when drilling at the opposite side. Thus, a recognition of this study is that it is better to place the marker tray as close to the osteotomy site as possible to make the process easier.

As for a navigation-guided implant surgery, good hand-eye coordination is of great importance. Operators need to stare at real-time images on the screen instead of the patient's oral cavity, and even an experienced surgeon can feel less confident at first. Therefore, a learning curve needs to be established. Arora et al. reported that a learning curve of cardio-thoracic and vascular surgical procedures was about 48 studies in total [22]. In this current study, an obvious learning curve was noticed from the first implant to the

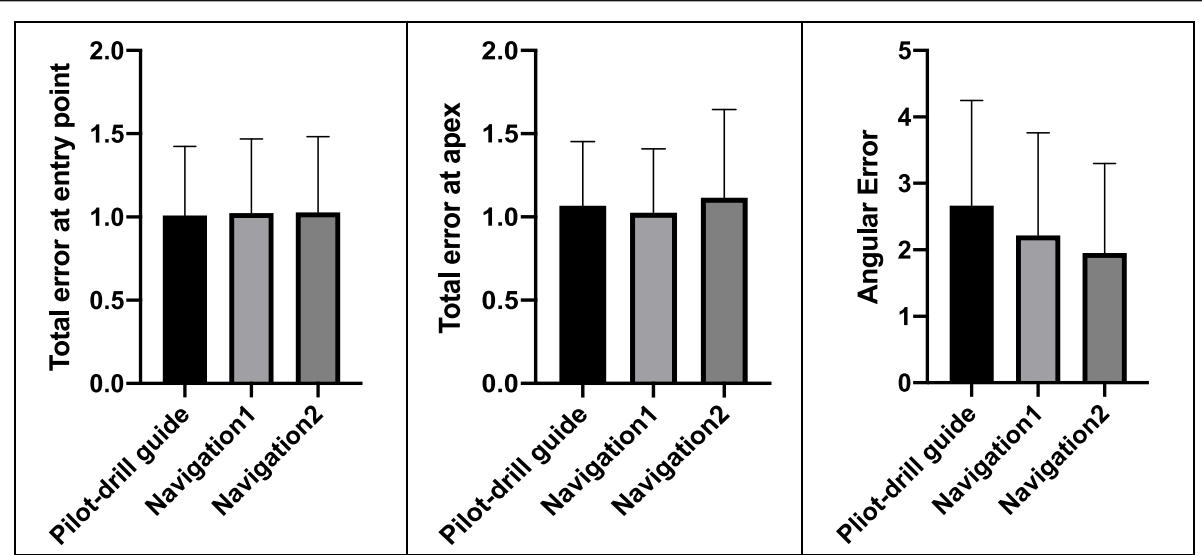

Fig. 8 Comparison of the three groups "pilot-drill guided," "marker tray in CBCT," and "3D-printed tray navigation" regarding the total error at entry point, the total error at apex, and the angular error 
twenty-fourth implant, so it can be suggested that no less than 25-30 osteotomies should be performed before surgeons treat a real patient with the system.

The most attracting advantage of navigation systems is visualization [23]. Tang et al. (2019) reported that anatomical structures that deviate from the norm can be reasons for greater inaccuracies in freehand implant placement [24]. Visualization of drill tips makes it possible for real-time correction, and more precise implant position should be promised theoretically. On the other hand, visualization of critical anatomical structures (floor of the sinus, mandibular canal, mental foramen, etc.) reduces the possibility of severe surgical complications. Besides that, navigation systems have more extensive indications compared to surgical guide systems. Navigation-guided implantation could also be more suitable for patients with limited mouth-opening or tight interdental space. As to non-experienced surgeons, the process can be easier with the help of navigation systems.

Despite their advantages in accuracy, it should be kept in mind that the handpiece used by the navigation systems should be occupied by a camera and its weight could influence the learned manipulation of the practitioner. In addition, the marker as an additional medium intraorally could also negatively influence the visual angle, which might lead to inaccuracies $[15,25]$. Besides that, the placement of the registration marker by edentulous patients remains still a clinical problem to be solved by the manufacturers.

Further in vitro studies with the Denacam System or equivalent systems must now provide information about the shown data. At least, newer planning software and planning methods enable a postoperative evaluation of the implant positions without having to rely to regular $\mathrm{x}$-rays because of the use of digital casts [26]. The accuracy of the implant placement is a very complex process that is prone to errors. Every single step from scanning, process planning, guide adjustment etc. influences the position of the implants [27]. Digital technologies can increase the accuracy, efficiency, and comfort of implant treatment and achieve satisfactory occlusal reconstruction results in patients with difficult anatomical conditions and complex cases $[28,29]$.

\section{Conclusions}

The accuracy of the evaluated navigation system was similar as the accuracy of a pilot-drill guide or other reported navigation systems. The accuracy of both preoperative workflows (marker tray in CBCT or 3Dprinted tray) was reliable enough for clinical use.

\section{Abbreviations}

CBCT: Cone beam computed tomography; PDG: Pilot-drill guide; 3dPTN: 3Dprinted tray navigation; MTCN: Marker tray in CBCT navigation; STL: Standard tessellation language

\section{Acknowledgements}

Not applicable.

\section{Authors' contributions}

FJ, CF, and EB performed the dynamic navigation and implant-guided surgeries. JS, FJ, CF, and AG analyzed and interpreted the collected data. HN and $J$ managed the literature search and collected the original literature. JS and FJ were the major contributors in writing the manuscript. CF and JW were the head of the project and developed the project. The authors read and approved the final manuscript.

\section{Funding}

This study was funded by the Christian-Albrechts-Universität zu Kiel (Forschungsförderung 2020-Junior F359929).

\section{Availability of data and materials}

The datasets used and/or analyzed during the current study are available from the corresponding author on reasonable request.

\section{Declarations}

Ethics approval and consent to participate Not applicable.

\section{Consent for publication}

All authors gave their consent for publication.

\section{Competing interests}

Johannes Spille, Feilu Jin, Eleonore Behrens, Yahya Açil, Jürgen Lichtenstein, Hendrik Naujokat, Aydin Gülses, Christian Flörke, and Jörg Wiltfang declare that they have no competing interests.

\section{Author details}

${ }^{1}$ Christian Albrechts University, Department of Oral and Maxillofacial Surgery, University Hospital of Schleswig-Holstein, Campus Kiel, Arnold-Heller-Straße 3, 24105 Kiel, Germany. ²Department of oral and Maxillofacial Surgery, School of Medicine, 2nd Affiliated Hospital of Zhejiang University, Hangzhou, Zhejiang, China

Received: 11 January 2021 Accepted: 17 March 2021

Published online: 30 April 2021

\section{References}

1. Wu D, Zhou L, Yang J, Zhang B, Lin Y, Chen J, et al. Accuracy of dynamic navigation compared to static surgical guide for dental implant placement. Int J Implant Dent. 2020;6(1):78. https://doi.org/10.1186/s40729-020-00272-0.

2. Canullo L, Tallarico M, Radovanovic S, Delibasic B, Covani U, Rakic M. Distinguishing predictive profiles for patient-based risk assessment and diagnostics of plaque induced, surgically and prosthetically triggered periimplantitis. Clin Oral Implants Res. 2016;27(10):1243-50. https://doi.org/1 $0.1111 / \mathrm{clr} .12738$

3. Vercruyssen $M$, Jacobs $R$, Van Assche $N$, van Steenberghe $D$. The use of $C$ scan based planning for oral rehabilitation by means of implants and its transfer to the surgical field: a critical review on accuracy. J Oral Rehabil. 2008;35(6):454-74. https://doi.org/10.1111/j.1365-2842.2007.01816.x.

4. Widmann G, Bale RJ. Accuracy in computer-aided implant surgery--a review. Int J Oral Maxillofac Implants. 2006;21(2):305-13.

5. Panchal N, Mahmood L, Retana A, Emery R 3rd. Dynamic navigation for dental implant surgery. Oral Maxillofac Surg Clin North Am. 2019;31(4):53947. https://doi.org/10.1016/j.coms.2019.08.001.

6. Siessegger M, Schneider BT, Mischkowski RA, Lazar F, Krug B, Klesper B, et al. Use of an image-guided navigation system in dental implant surgery in anatomically complex operation sites. J Craniomaxillofac Surg. 2001;29(5): 276-81. https://doi.org/10.1054/jcms.2001.0242. 
7. Emery RW, Merritt SA, Lank K, Gibbs JD. Accuracy of dynamic navigation for dental implant placement-model-based evaluation. J Oral Implantol. 2016; 42(5):399-405. https://doi.org/10.1563/aaid-joi-D-16-00025.

8. Reyes A, Turkyilmaz I, Prihoda TJ. Accuracy of surgical guides made from conventional and a combination of digital scanning and rapid prototyping techniques. J Prosthet Dent. 2015;113(4):295-303. https://doi.org/10.1016/j. prosdent.2014.09.018.

9. Park SJ, Leesungbok R, Cui T, Lee SW, Ahn SJ. Reliability of a CAD/CAM surgical guide for implant placement: an in vitro comparison of surgeons' experience levels and implant sites. Int J Prosthodont. 2017;30(4):367-169. https://doi.org/10.11607/ijp.5179.

10. Bell CK, Sahl EF, Kim YJ, Rice DD. Accuracy of implants placed with surgical guides: thermoplastic versus 3D printed. Int J Periodontics Restorative Dent. 2018;38(1):113-9. https://doi.org/10.11607/prd.3254.

11. Gargallo-Albiol J, Barootchi S, Salomo-Coll O, Wang HL. Advantages and disadvantages of implant navigation surgery. A systematic review. Ann Anat. 2019;225:1-10. https://doi.org/10.1016/j.aanat.2019.04.005.

12. Noharet R, Pettersson A, Bourgeois D. Accuracy of implant placement in the posterior maxilla as related to 2 types of surgical guides: a pilot study in the human cadaver. J Prosthet Dent. 2014;112(3):526-32. https://doi.org/10.101 6/j.prosdent.2013.12.013

13. Kim SG, Lee WJ, Lee SS, Heo MS, Huh KH, Choi SC, et al. An advanced navigational surgery system for dental implants completed in a single visit: an in vitro study. J Craniomaxillofac Surg. 2015;43(1):117-25. https://doi. org/10.1016/j.jcms.2014.10.022.

14. Amin S, Weber HP, Finkelman M, El Rafie K, Kudara Y, Papaspyridakos P. Digital vs. conventional full-arch implant impressions: a comparative study. Clin Oral Implants Res. 2017;28(11):1360-7. https://doi.org/10.1111/clr.12994.

15. Chen CK, Yuh DY, Huang RY, Fu E, Tsai CF, Chiang CY. Accuracy of implant placement with a navigation system, a laboratory guide, and freehand drilling. Int J Oral Maxillofac Implants. 2018;33(6):1213-8. https://doi.org/1 $0.11607 /$ jomi.6585.

16. Jorba-Garcia A, Figueiredo R, Gonzalez-Barnadas A, Camps-Font O, Valmaseda-Castellon E. Accuracy and the role of experience in dynamic computer guided dental implant surgery: an in-vitro study. Med Oral Patol Oral Cir Bucal. 2019;24(1):e76-83. https://doi.org/10.4317/medoral.22785.

17. Sun TM, Lan TH, Pan CY, Lee HE. Dental implant navigation system guide the surgery future. Kaohsiung J Med Sci. 2018;34(1):56-64. https://doi.org/1 0.1016/j.kjms.2017.08.011.

18. Stefanelli LV, DeGroot BS, Lipton DI, Mandelaris GA. Accuracy of a dynamic dental implant navigation system in a private practice. Int J Oral Maxillofac Implants. 2019;34(1):205-13. https://doi.org/10.11607/jomi.6966.

19. Jung RE, Schneider D, Ganeles J, Wismeijer D, Zwahlen M, Hammerle CH, et al. Computer technology applications in surgical implant dentistry: a systematic review. Int J Oral Maxillofac Implants. 2009;24(Suppl):92-109.

20. Abduo J, Lau D. Accuracy of static computer-assisted implant placement in anterior and posterior sites by clinicians new to implant dentistry: in vitro comparison of fully guided, pilot-guided, and freehand protocols. Int J Implant Dent. 2020;6(1):10. https://doi.org/10.1186/s40729-020-0205-3.

21. Mozer PS. Accuracy and deviation analysis of static and robotic guided implant surgery: a case study. Int J Oral Maxillofac Implants. 2020;35(5):e8690. https://doi.org/10.11607/jomi.8231.

22. Arora KS, Khan N, Abboudi H, Khan MS, Dasgupta P, Ahmed K. Learning curves for cardiothoracic and vascular surgical procedures--a systematic review. Postgrad Med. 2015;127(2):202-14. https://doi.org/10.1080/003254 81.2014.996113

23. Block MS, Emery RW, Lank K, Ryan J. Implant placement accuracy using dynamic navigation. Int J Oral Maxillofac Implants. 2017;32(1):92-9. https:// doi.org/10.11607/jomi.5004

24. Tang T, Huang Z, Liao L, Gu X, Zhang J, Zhang X. Factors that influence direction deviation in freehand implant placement. J Prosthodont. 2019; 28(5):511-8. https://doi.org/10.1111/jopr.13065.

25. Lopes A, de Araujo NM, Santos D. The workflow of a new dynamic navigation system for the insertion of dental implants in the rehabilitation of edentulous Jaws: Report of Two Cases. J Clin Med. 2020;9(2). https://doi. org/10.3390/jcm9020421.

26. Liu Y, Ye H, Wang S, Zhang L, Zhou Y. An open protocol for evaluating the accuracy of guided implant surgery by using digital casts. J Prosthet Dent. 2020. https://doi.org/10.1016/j.prosdent.2020.05.039.
27. Abduo J, Lau D. Effect of manufacturing technique on the accuracy of surgical guides for static computer-aided implant surgery. Int J Oral Maxillofac Implants. 2020;35(5):931-8. https://doi.org/10.11607/jomi.8186.

28. Liang C, Lin X, Li J, Geng W. A digital workflow for implant treatment and occlusal reconstruction in a patient with an edentulous mandible: a case history report. Int J Prosthodont. 2020;33(5):565-71. https://doi.org/10.11 607/ijp.6614.

29. Apostolakis D, Kourakis G. CAD/CAM implant surgical guides: maximum errors in implant positioning attributable to the properties of the metal sleeve/osteotomy drill combination. Int J Implant Dent. 2018;4(1):34. https:// doi.org/10.1186/s40729-018-0146-2.

\section{Publisher's Note}

Springer Nature remains neutral with regard to jurisdictional claims in published maps and institutional affiliations.

\section{Submit your manuscript to a SpringerOpen ${ }^{\circ}$ journal and benefit from:}

- Convenient online submission

- Rigorous peer review

- Open access: articles freely available online

High visibility within the field

- Retaining the copyright to your article

Submit your next manuscript at $\boldsymbol{\nabla}$ springeropen.com 